\title{
Cognitive Load Theory: New Conceptualizations, Specifications, and Integrated Research Perspectives
}

\author{
Fred Paas • Tamara van Gog • John Sweller
}

Published online: 6 May 2010

(C) The Author(s) 2010. This article is published with open access at Springerlink.com

\begin{abstract}
Over the last few years, cognitive load theory has progressed and advanced rapidly. The articles in this special issue, which document those advances, are based on contributions to the 3rd International Cognitive Load Theory Conference (2009), Heerlen, The Netherlands. The articles of this special issue on cognitive load theory discuss new conceptualizations of the different categories of cognitive load, an integrated research perspective of process-oriented and cognitive load approaches to collaborative learning, an integrated research perspective of cognitive and social-cognitive approaches to examplebased learning, and a specification of the theory focusing on the acquisition of generalized knowledge structures as a means to facilitate flexible problem-solving skills. This article provides a short introduction to the theory, discusses some of its recent advances, and provides an overview of the contributions to this issue.
\end{abstract}

Keywords Cognitive load theory · Working memory · Integrated research perspectives

During the past two decades, cognitive load theory (CLT: Paas et al. 2003a, 2004; Sweller 1988; Sweller et al. 1998; Van Merriënboer and Sweller 2005) has become an influential theory in the fields of educational psychology and instructional design. Evidence for that influence comes from Ozcinar (2009), who, examining research publications and trends in instructional design during the period 1980-2008, found that 'cognitive load theory' was

F. Paas · T. van Gog

Institute of Psychology, Erasmus University Rotterdam, Rotterdam, The Netherlands

F. Paas

CELSTEC, Open Universiteit Nederland, Heerlen, The Netherlands

J. Sweller

School of Education, University of New South Wales, Sydney, Australia

F. Paas $(\bowtie)$

Department of Psychology, Erasmus University Rotterdam, P.O. Box 1738, 3000 DR Rotterdam,

The Netherlands

e-mail: Paas@fsw.eur.nl 
the second most frequently used phrase. Six of the top ten most cited articles were concerned with CLT. In addition, Jones et al. (2010) investigated individual productivity in the top five educational psychology journals from 2003 to 2008 . Four of the top 20 most productive researchers they identified use cognitive load theory as a central theory in their work.

There are both scientific and practical reasons why CLT has survived and become influential. Firstly, from a scientific point of view, the theory has evolved by withstanding rigorous tests of falsification, consistent confirmation of existing hypotheses, timely modifications of the theory as required by new data, and generation of new hypotheses. For example, P. A. Kirschner et al. (2006) have shown that there exists a clear body of research using controlled experiments indicating that the relatively guided instructional procedures supported by cognitive load theorists are more effective (at least for novice students) than relatively unguided instructional procedures used by advocates of discovery or inquiry learning. Secondly, again from a scientific perspective, the theory has been responsive to the challenges of contemporary education by building on theories and research results from other disciplines, such as evolutionary biology (e.g., Sweller 2003, 2004; Sweller and Sweller 2006) and neuroscience (e.g., Kirschner et al. 2009a; Van Gog et al. 2009). Thirdly, from a practical point of view, the theory has generated over the last two decades a unique variety of useful and effective instructional designs and procedures (for overviews, see Sweller et al. 1998; Van Merriënboer and Sweller 2005).

The articles in this special issue continue the procedures used by CLT. They discuss new conceptualizations of the different categories of cognitive load, an integrated perspective of process-oriented and cognitive load approaches to collaborative learning, an integrated perspective of cognitive and social-cognitive approaches to example-based learning, and a specification of the theory focusing on the acquisition of generalized knowledge structures as a means to facilitate flexible problem-solving skills. This article starts with a short introduction to CLT, then discusses some of its recent advances, and finally provides an overview of the contributions to this issue.

Cognitive load theory is concerned with the learning of complex cognitive tasks, in which learners are often overwhelmed by the number of interactive information elements that need to be processed simultaneously before meaningful learning can commence. Instructional control of the excessively high load imposed by complex tasks provides the focus of CLT. To realize this control, CLT uses current knowledge about the human cognitive architecture to generate instructional techniques. This architecture consists of an effectively unlimited long-term memory (LTM), which interacts with a working memory (WM) that is very limited in both capacity and duration. LTM stores previously acquired information. WM processes information either prior to it being stored in LTM or after it has been stored. It can be equated with consciousness, in the sense that we are only conscious of the information currently being processed in WM and are oblivious of the far larger amount of information stored in LTM.

LTM is viewed as the central structure of human cognition. It contains huge amounts of knowledge that can be described as hierarchically organized schemas that allow us to categorize different problem states and decide upon the most appropriate solution moves. The extent of knowledge held in LTM determines the level of performance by an individual in a given area. Accordingly, how the large store of knowledge held in LTM is best acquired provides the central concern of CLT. The theory assumes that most knowledge does not have to be gained from experience but can be borrowed from other people (which has an obvious evolutionary advantage) and thus it follows that cognitive load theorists spend much of their time considering how that knowledge should be structured when it is 
presented to learners, as well as in which activities learners should engage when acquiring information.

How knowledge should be presented to learners and the activities in which they should engage depends on the characteristics of WM because WM first processes information before it can be stored in LTM. WM has two well-known characteristics: when processing novel information, it is very limited in both duration (Peterson and Peterson 1959) and capacity (Baddeley 1992; Miller 1956). With regard to duration, almost all information stored in WM and not rehearsed is lost within $30 \mathrm{~s}$. The processing capacity of WM is limited to only $4 \pm 1$ element (Cowan 2001). This limitation is necessary because when dealing with novel elements during problem solving, for which full knowledge is unavailable, there is no alternative to a random generate-and-test procedure in which it is considered how various elements should be combined. A large number of elements would result in combinatorial explosions preventing adequate processing (Sweller and Sweller 2006).

The interactions between WM and LTM may be even more important than the processing limitations. The limitations of WM only apply to new, yet to be learned information. Previously organized and stored information in LTM is not prone to combinatorial explosions, and thus, the limitations that must be imposed on novel information do not apply to familiar information. While novel information only can be stored for brief periods with severe limitations on the amount of such information, when dealing with previously learned information stored in LTM, these limitations disappear. In the sense that very large amounts of information can be brought back from LTM to WM over indefinite periods of time, the capacity and temporal limits of WM become irrelevant for information that has already been acquired.

One aim of CLT-based instruction is to ensure that learners' WM is not overloaded by the information presented. A related and, in some sense, ultimate aim of instruction is that learners acquire sufficient information in LTM to ensure that they can function without the debilitating limitations of WM when dealing with novel information.

This cognitive architecture suggests the issues that instructional designers should consider. Instruction most frequently deals with information that is novel for learners. WM limitations apply to that information, and hence, it is the structure of that information that must be carefully considered. CLT identifies three categories of cognitive load: intrinsic, extraneous, and germane (Paas et al. 2003b; Sweller et al. 1998). Intrinsic load is the load caused by the complexity of the materials to be learned and, thus, the complexity of the schemas that must be acquired. Germane load refers to the WM resources required to deal with intrinsic cognitive load. Extraneous load is the load caused by poorly designed instructional procedures that interfere with schema acquisition. Intrinsic load is dependent upon element interactivity (Sweller and Chandler 1994), the number of elements that need to be processed simultaneously by the learner. If element interactivity is high, learning becomes difficult and WM-resource intensive, whereas for low element interactivity material, learning is easier, requiring fewer WM resources. When instructional material is poorly constructed, extraneous load is generated because the learner is diverted away from schema acquisition and uses up precious WM resources by trying to deal with a suboptimal learning environment (Sweller et al. 1998). Because intrinsic and extraneous cognitive load are additive, an increase in extraneous cognitive load reduces the WM resources available to deal with intrinsic cognitive load and hence reduces germane cognitive load. Decreasing extraneous cognitive load frees resources to deal with intrinsic cognitive load and thus increases germane cognitive load. When intrinsic cognitive load is high, it becomes important to decrease extraneous cognitive load; otherwise, the combination of both might 
exceed the maximum cognitive capacity and thus prevent effective, or germane, processing activities to occur. From an instructional design point of view, it is important to consider extraneous and germane cognitive load as communicating vessels, because the reduction of extraneous cognitive load can free cognitive resources for an increase in germane cognitive load (Paas et al. 2003a, b).

To manage the WM load and facilitate the changes in LTM associated with learning, first of all, extraneous load must be eliminated. Studying worked examples (instead of solving conventional problems) has been identified as an effective way of reducing extraneous load, because the learner can devote all available WM capacity to studying a worked-out solution and constructing a schema for solving similar problems in LTM (e.g., Paas 1992; Paas and Van Gog 2006) However, freeing WM capacity by eliminating extraneous load is not a sufficient technique for instructional conditions to be effective. Intrinsic load must be managed in such a way that the simultaneous processing of all interactive information elements (germane cognitive load) is possible. A very heavy intrinsic cognitive load may exceed WM capacity and thus prevent processing of all elements. One way to manage intrinsic load is by applying a so-called part-whole approach, in which the number of information elements and interactions between elements is initially reduced by simplifying the tasks, after which more and more elements and interactions are added (e.g., Van Merriënboer et al. 2006). As more elements are added, germane cognitive load can increase without exceeding WM capacity. Other effective ways to increase germane load are by increasing the variability of learning tasks (e.g., Paas and Van Merriënboer 1994) or asking learners to self-explain how they arrived at a problem solution (e.g., Renkl 2002). In both cases, the nature of the task is changed in a manner that appropriately increases intrinsic cognitive load and thus increases the WM resources required, resulting in increases in germane cognitive load.

The first article in this issue, by Sweller (this issue), is responsive to recent discussions regarding problems of defining and measuring the different categories of load postulated by CLT (e.g., Schnotz and Kürschner 2007). The article provides a more uniform foundation for the division of cognitive load into categories. It redefines the constructs of extraneous and germane cognitive load by relating them to element interactivity and analyzes the consequences of explaining the various cognitive load effects in terms of element interactivity. Element interactivity was originally only used as a defining characteristic of intrinsic cognitive load (Sweller 2004; Sweller and Chandler 1994). Extraneous cognitive load is now assumed to be caused by element interactivity not essential to the task at hand, which can be eliminated by altering instructional procedures. Germane load is now assumed to consist of the WM resources used to deal with element interactivity that contributes to learning. Sweller argues that by defining both intrinsic and extraneous cognitive load in terms of element interactivity, it may be possible to analyze element interactivity prior to an experiment and thus more easily predict experimental outcomes. According to Sweller, the new conceptualizations might have considerable theoretical and practical advantages. From a theoretical perspective, relations between intrinsic, extraneous, and germane cognitive load are provided with a more rational foundation. From a practical point of view, the new conceptualizations could lead to the generation of new instructional procedures.

The second article by Janssen et al. (this issue) builds on a recent line of CLT research that recognizes collaborative learning as an alternative way of overcoming individual WM limitations (F. Kirschner et al. 2009a, b). Until recently, cognitive load research was concerned exclusively with designing, developing, and researching instructional techniques for managing the cognitive load imposed by complex cognitive learning tasks in individual 
learning settings. According to the CLT perspective, a group of collaborative learners can be considered as an information processing system (Hinsz et al. 1997), consisting of multiple, limited WMs that can create a collective working space. In collaborative learning settings, the cognitive load associated with carrying out a learning task can be distributed across those multiple collaborating working memories, which reduces the risk of overloading each group member and creates a larger reservoir of cognitive resources. F. Kirschner et al. (2009b) have shown that because of the distribution advantage and expanded processing capacity, meaningful learning from tasks that impose a high cognitive load is more likely to occur in a collaborative learning setting than in an individual learning setting.

Whereas cognitive load research on collaborative learning can reveal the load imposed upon group members during collaboration, process-oriented research can be used to uncover the mechanisms involved in collaboration that impose this load. Janssen et al. discuss how the combination of process-oriented and cognitive load approaches might lead to a deeper understanding of learning through collaboration, more specifically, of the relationships between the antecedents of collaboration, the collaboration process itself, and the consequences of collaborative learning.

The third article by Van Gog and Rummel (this issue) provides a review, comparison, and contrast of learning from worked examples and modeling examples. Whereas worked examples are investigated in cognitive load research and require learners to study didactical worked-out problem solutions in written format, modeling examples are investigated in social-cognitive research and require learners to observe someone performing a task, and this model may be behaving didactically or naturally (making and correcting errors during performance). Since these two forms of learning from examples originate from different academic disciplines, they are not typically explored in the same empirical studies or discussed in the same papers, even though they have clear and interesting connections and analogs. They also have some clear differences, though. For example, whereas studies on modeling examples do not tend to consider cognitive load involved and might benefit from doing so, these studies often do consider other measures that are not typically considered but might be interesting for cognitive load research on worked examples, such as effects on students' self-efficacy. In addition, these studies on modeling examples have also addressed the effects of model characteristics such as age, gender, and type of performance (e.g., mastery vs. coping), which are not relevant for didactical, written text-based worked examples, but may be relevant in video-based examples or animations that are increasingly being used as alternatives to written text-based worked examples. The article makes clear that studies drawing on both perspectives may advance our knowledge base on examplebased learning.

The fourth article by Kalyuga et al. (this issue) suggests that CLT could be improved by placing an increased emphasis on generalized knowledge structures as opposed to its current emphasis on domain-specific knowledge. The success of CLT derives to a large extent from its emphasis on domain-specific knowledge, and in fact, it could be argued that CLT was devised in part as a reaction to the failure of many other theories advocating the acquisition of general knowledge structures. However, this article does not focus on those generic universal problem-solving heuristics but on domain-specific knowledge occupying a medium level of generality between generic knowledge and the routine low-level knowledge structures directly associated with specific problem situations. Based on a review of theoretical foundations and empirical evidence on CLT, problem solving, and adaptive instruction, the authors conclude that generalized knowledge structures could also be important for developing flexible expertise. 
In conclusion, we believe that the papers of this issue attest to the continuing strength and usability of CLT. For many years, the theory has provided a source of novel, instructional design procedures. The current papers provide evidence that it is capable of continuing to do so.

Open Access This article is distributed under the terms of the Creative Commons Attribution Noncommercial License which permits any noncommercial use, distribution, and reproduction in any medium, provided the original author(s) and source are credited.

\section{References}

Baddeley, A. (1992). Working memory. Science, 255, 556-559.

Cowan, N. (2001). The magical number 4 in short-term memory: A reconsideration of mental storage capacity. The Behavioral and Brain Sciences, 24, 87-114.

Hinsz, V. B., Tindale, R. S., \& Vollrath, D. A. (1997). The emerging conceptualization of groups as information processors. Psychological Bulletin, 121, 43-64.

Janssen, J., Kirschner, F., Erkens, G., Kirschner, P. A., \& Paas, F. (this issue). Making the black box of collaborative learning transparent: Combining process-oriented and cognitive load approaches. Educational Psychology Review.

Jones, S. J., Fong, C. J., Torres, L. G., Yoo, J. H., Decker, M. L., \& Robinson, D. H. (2010). Productivity in educational psychology journals from 2003-2008. Contemporary Educational Psychology, 35, 11-16.

Kalyuga, S., Renkl, A., \& Paas, F. (this issue). Facilitating flexible problem solving: A cognitive load perspective. Educational Psychology Review.

Kirschner, P. A., Sweller, J., \& Clark, R. E. (2006). Why minimal guidance during instruction does not work: An analysis of the failure of constructivist, discovery, problem-based, experiential, and inquiry-based teaching. Educational Psychologist, 41, 75-86.

Kirschner, F., Paas, F., \& Kirschner, P. A. (2009a). A cognitive load approach to collaborative learning: United brains for complex tasks. Educational Psychology Review, 21, 31-42.

Kirschner, F., Paas, F., \& Kirschner, P. A. (2009b). Effects of individual and group-based learning from complex cognitive tasks on efficiency of retention and transfer performance. Computers in Human Behavior, 25, 306-314.

Miller, G. A. (1956). The magical number seven, plus or minus two: Some limits on our capacity for processing information. Psychological Review, 63, 81-97.

Ozcinar, Z. (2009). The topic of instructional design in research journals: A citation analysis for the years 1980-2008. Australasian Journal of Educational Technology, 25, 559-580.

Paas, F. (1992). Training strategies for attaining transfer of problem-solving skill in statistics: A cognitiveload approach. Journal of Education \& Psychology, 84, 429-434.

Paas, F., \& Van Gog, T. (2006). Optimising worked example instruction: Different ways to increase germane cognitive load. Learning and Instruction, 16, 87-91.

Paas, F., \& Van Merriënboer, J. J. G. (1994). Variability of worked examples and transfer of geometrical problem-solving skills: A cognitive-load approach. Journal of Education \& Psychology, 86, 122-133.

Paas, F., Renkl, A., \& Sweller, J. (2003a). Cognitive load theory and instructional design: Recent developments. Educational Psychologist, 38, 1-4.

Paas, F., Tuovinen, J. E., Tabbers, H., \& Van Gerven, P. W. (2003b). Cognitive load measurement as a means to advance cognitive load theory. Educational Psychologist, 38, 63-71.

Paas, F., Renkl, A., \& Sweller, J. (2004). Cognitive load theory: Instructional implications of the interaction between information structures and cognitive architecture. Instructional Science, 32, 1-8.

Peterson, L., \& Peterson, M. J. (1959). Short-term retention of individual verbal items. Journal of Experimental Psychology, 58, 193-198.

Renkl, A. (2002). Worked-out examples: Instructional explanations support learning by self-explanations. Learning and Instruction, 12, 529-556. 
Schnotz, W., \& Kürschner, C. (2007). A reconsideration of cognitive load theory. Educational Psychology Review, 19, 469-508.

Sweller, J. (1988). Cognitive load during problem solving: Effects on learning. Cognitive Science, 12, 257285.

Sweller, J. (2003). Evolution of human cognitive architecture. In B. Ross (Ed.), The psychology of learning and motivation (Vol. 43, pp. 215-266). San Diego: Academic Press.

Sweller, J. (2004). Instructional design consequences of an analogy between evolution by natural selection and human cognitive architecture. Instructional Science, 12, 185-233.

Sweller, J. (this issue). Element interactivity and intrinsic, extraneous and germane cognitive load. Educational Psychology Review.

Sweller, J., \& Chandler, P. (1994). Why some material is difficult to learn. Cognition and Instruction, 12, $185-233$.

Sweller, J., \& Sweller, S. (2006). Natural information processing systems. Evolutionary Psychology, 4, 434458.

Sweller, J., Van Merriënboer, J. J. G., \& Paas, F. (1998). Cognitive architecture and instructional design. Educational Psychology Review, 10, 251-295.

Van Gog, T., \& Rummel, N. (this issue). Example-based learning: Integrating cognitive and social-cognitive research perspectives. Educational Psychology Review.

Van Gog, T., Paas, F., Marcus, N., Ayres, P., \& Sweller, J. (2009). The mirror-neuron system and observational learning: Implications for the effectiveness of dynamic visualisations. Educational Psychology Review, 21, 21-30.

Van Merriënboer, J. J. G., \& Sweller, J. (2005). Cognitive load theory and complex learning: Recent developments and future directions. Educational Psychology Review, 17, 147-178.

Van Merriënboer, J. J. G., Kester, L., \& Paas, F. (2006). Teaching complex rather than simple tasks: Balancing intrinsic and germane load to enhance transfer of learning. Applied Cognitive Psychology, 20, 343-352. 